\title{
Hail Sensing Probes: Feasibility Analysis for Probes to Monitor and Study Hail
}

\author{
Silvano Bertoldo, Claudio Lucianaz, Marco Allegretti \\ CINFAI (Interuniversity National Consortium for the Physics of Atmosphere and Hydrosphere), Politecnico di \\ Torino, Torino, Italy \\ Email: silvano.bertoldo@polito.it
}

Received 7 January 2016; accepted 5 March 2016; published 8 March 2016

Copyright (C) 2016 by authors and Scientific Research Publishing Inc.

This work is licensed under the Creative Commons Attribution International License (CC BY). http://creativecommons.org/licenses/by/4.0/

(c) (i) Open Access

\begin{abstract}
This work presents the feasibility study regarding the realization of hail sensing probes for remote sensing and study of hail. The probes are designed as artificial hailstones in order to study both the physical properties of the portion of atmosphere where the formation of hail occurs and the modification of atmospheric conditions while the hailstones are falling to the ground. The basic idea is to realize sensors with a similar fluid-dynamic behavior with respect to hailstones; the density, the weight and the size of the probes are determined. Consequently, the specification of the electronic boards, sensors, and material to realize the probes are studied and presented. The hail sensing probes can be dropped by a plane, or potentially a UAV (Unmanned Aircraft Vehicle), which fly above and through the clouds where the hail formation occurs. During their falling to the ground, the sensors installed on the probe measure directly different parameters such as humidity, temperature, pressure, acceleration. All data are sent to a receiver located on the ground. The study of the sampling frequency of each sensor is presented, together with the analysis of the propagation channel, in order to assure a robust communication link between probes and the ground receiver. The energy balance is also computed. The work demonstrates that a set of this kind of disposable sensors can be realized. They can be used for efficient monitoring operations and studies of hail formation dynamics and conditions, thus increasing the set of instruments for the monitoring and remote sensing of hail.
\end{abstract}

\section{Keywords}

Hail, Artifical Hailstones, Sensing Probes, Hail Studies, Hail Sensors, Sensors, UAV

\section{Introduction}

The remote sensing of hail has always been mainly focused on post-event analysis and assessment of crop 
damages. These researches are usually made after intense hailstorm events and are supported by insurance and agricultural institutions; they are based on data and information coming from different sources and techniques, from SAR to hyperspectral images, to photogrammetry, to climatological studies and so on.

To employ remote sensing techniques to study the hail formation, the hail properties and characteristics and to investigate the atmospheric dynamics that can lead to the formation of hailstones directly where it happens is less common. Only a few studies have been proposed using dual linear radar (e.g. in [1] [2]). The most recent polarimetric Doppler radars allow to discern between rain and hail even during severe thunderstorm, but they are not widely used to especially study hail. To record and study hailstorms, other studies developed specific sensors similar to rain gauges [3], or they try to implement innovative and uncommon techniques, such as, for instance, using piezoelectric microphone [4] to record hail and establish the intensity.

Since 1970s the studies of tornadoes give some possibilities to realize probes to send directly inside them to collect measurements of different parameters (e.g. wind). Some preliminary studies and designs were given in the early seventies [5]. Nowadays these ideas are the basis of different research activities and industrial research projects. Among them, very interesting is the "Tornado Probe Idea \& Concept" proposed by WXOpenSource.org, an open weather research technology community to facilitated iscussion, design and deployment of new systems for meteorological purposes [6].

Considering the lack of specific probes to study the hail formation directly inside the clouds where the hailstorm formation occurs, and on the basis of the knowledge related to probes to study the tornadoes, the present work shows an early feasibility analysis of a set of disposable hailstone sensing probes that can be dropped directly where a hailstorm is going to happen. The probes can be dropped using an aircraft or even a UAV (Unmanned Aircraft Vehicle) which can fly high enough to be over the portion of atmosphere where the hail forms. They are designed with the most similar possible fluid-dynamic properties of hailstones. While they are falling to the ground, they acquire data and send them to a receiver located on the ground which stores information for post processing operations and analysis.

In the following, after a brief but necessary analysis of hail characteristics, both a theoretical analysis and practical feasibility study of such probes are presented.

\section{Hail Characteristics}

Hail is a form of solid meteorological precipitation. It is distinct from sleet, although the two are often confused for one another. It consists of balls or irregular lumps of ice, each of which is called a hailstone. Sleet falls generally in cold weather while hail growth is greatly inhibited at cold temperatures [7].

The hail formation is possible within most thunderstorms and requires environments of strong, upward motion of air, lowered heights of the freezing level and very high atmospheric humidity. At middle latitudes it is more likely that hailstorms occur in summer and are most common within continental interiors. Hail formation is considerably more likely when the freezing level is below the altitude of about $3500 \mathrm{~m}$. The movement of dry air into strong thunderstorms over continents can increase the frequency of hail. The evaporation cooling lowers the freezing level of clouds, thus giving hail a larger volume to grow in. Around a thunderstorm, hail formation is most likely within the cloud at the elevation of $6000 \mathrm{~m}$, despite its formation altitude can reach up to 11,000 m [8].

A hail storm generally has a duration that rarely exceeds 15 minutes. During a hailstorm, and immediately after, the ground temperature is lowered rapidly (it is possible down to $10^{\circ} \mathrm{C}$ in half an hour) because the solid ice subtracts heat to the environment to turn into liquid water.

\section{Hailstone Characteristics}

Since the objective of the work is to study the feasibility analysis of a sensor with fluid-dynamics characteristics similar to a hailstone, it is important to analyze them.

Depending on the way of its formation, each hailstone can reach a spherical size with a diameter from a minimum value of $d_{\min }=5 \mathrm{~mm}$ to a maximum $d_{\max }=15 \mathrm{~cm}$. Hailstones with a diameter of less than $5 \mathrm{~mm}$ are properly defined as ice pellets [6].

The density of hailstones and ice pellets was measured several times using different techniques. In 1958 [9] a first attempts to measure the density of ice pellets gave a density value $\rho=0.5-0.7 \mathrm{~g} / \mathrm{cm}^{3}$. In 1963 a work of Braham [10] provided a density values $\rho=0.87-0.89 \mathrm{~g} / \mathrm{cm}^{3}$. Then, Prodi [11] and List [12] in 1970 obtained 
separately, with 2 different techniques, a density of hailstones $\rho=0.82-0.87 \mathrm{~g} / \mathrm{cm}^{3}$. Various other measures were carried by other scientists giving values that are not much different. A good panoramic is reported in a work of Knight [13] and in a more recent paper of Castellano et al. [14]. Taking into account all the measured density value, in the present work we consider an average density of hailstones $\rho=0.87 \mathrm{~g} / \mathrm{cm}^{3}$.

To study the fluid-dynamics behavior of an object it is important to know its terminal velocity. The terminal velocity is the highest theoretical velocity that a body immersed in a fluid can reach when subjected to a force of flow resistance due to the presence of the fluid itself, which exactly compensates forces acting in the opposite way. It can be evaluated as in (1), where $m$ is the mass of the object, $g$ is the acceleration due to the gravity, $\rho$ is the density of the fluid through which the object is falling, $S$ is the projected contact surface of the object and $C_{d}$ is the drag coefficient.

$$
V_{l}=\sqrt[2]{\frac{2 m g}{\rho S C_{d}}}
$$

Three different spherical hailstones (with common value $C_{d}=0.43$ for spherical objects) are considered, with a diameter equal to $d_{1}=5 \mathrm{~mm}, d_{2}=10 \mathrm{~mm}$ and $d_{3}=15 \mathrm{~mm}$. The air density is then assumed to be equal to $\rho_{\text {air }}=$ $1.2 \mathrm{~kg} / \mathrm{m}^{3}\left(\rho_{\text {air }}=1200 \mathrm{~g} / \mathrm{m}^{3}\right)$ in proximity of the ground and equal to $\rho_{\text {air }}=413 \mathrm{~g} / \mathrm{m}^{3}$ at an altitude of $10,000 \mathrm{~m}$. Table 1 reports projected surface, volume, mass, and the terminal velocity values of such hailstones.

\section{Hail Sensing Probes: Theoretical Feasibility}

In order to have hailstones and artificial probes designed with the same fluid-dynamics properties, it is fundamental that they have the same terminal velocity: $V_{\text {hail }}=V_{\text {probe }}$. This must be ensured considering the mass of the printed circuit board (PCB), of the all required components and integrated circuits, of the battery, of the radiofrequency transceiver, and of the outer shell.

Therefore, the following equation (2) must be satisfied. The left term is related to hail while the right one related to a single probe. The terms $\Delta_{\text {hail }}$ and $\Delta_{\text {probe }}$ contain all the effects that may affect the fall of the object:

$$
\sqrt[2]{\frac{2 m_{\text {hail }} g}{\rho_{\text {air }} S_{\text {hail }} \Delta_{\text {hail }}}}=\sqrt[2]{\frac{2 m_{\text {probe }} g}{\rho_{\text {air }} S_{\text {probe }} \Delta_{\text {probe }}}}
$$

Spherical hailstone and probe are considered with radius equal to $R_{\text {hail }}$ and $R_{\text {probe }}$ respectively. It is possible to evaluate the mass of both of them by knowing their densities. Note that the $\rho_{\text {probe }}$ is an equivalent density considering together both all the electronic equipment and the container.

$$
\begin{gathered}
m_{\text {hail }}=\frac{4}{3} \pi R_{\text {hail }}^{3} \rho_{\text {hail }} \\
m_{\text {probe }}=\frac{4}{3} \pi R_{\text {probe }}^{3} \rho_{\text {probe }}
\end{gathered}
$$

\begin{tabular}{|c|c|c|c|c|c|c|}
\hline \multirow{2}{*}{ Altitude $[\mathrm{m}]$} & \multicolumn{6}{|c|}{ Hailstone characteristics (density $\rho=0.87 \mathrm{~g} / \mathrm{cm}^{3}$ ) } \\
\hline & Diameter [mm] & Surface $\left[\mathrm{m}^{2}\right]$ & Volume $\left[\mathrm{m}^{3}\right]$ & Mass [g] & $\begin{array}{c}\text { Theoretical Terminal } \\
\text { Velocity }[\mathrm{m} / \mathrm{s}]\end{array}$ & $\begin{array}{l}\text { Theoretical Terminal } \\
\text { Velocity }[\mathrm{km} / \mathrm{h}]\end{array}$ \\
\hline 10,000 & \multirow{2}{*}{5} & \multirow{2}{*}{$2.6 \mathrm{e}-5$} & \multirow{2}{*}{$6.5 \mathrm{e}-8$} & \multirow{2}{*}{0.06} & 16 & $\sim 58$ \\
\hline Ground & & & & & 9.3 & $\sim 33$ \\
\hline 10,000 & \multirow{2}{*}{10} & \multirow{2}{*}{$1 e^{-4}$} & \multirow{2}{*}{$5 e-6$} & \multirow{2}{*}{0.4} & 21 & $\sim 76$ \\
\hline Ground & & & & & 12.3 & $\sim 45$ \\
\hline 10,000 & \multirow{2}{*}{15} & \multirow{2}{*}{0.02} & \multirow{2}{*}{$1.77 \mathrm{e}-3$} & \multirow{2}{*}{1540} & 92 & $\sim 330$ \\
\hline Ground & & & & & 54 & $\sim 190$ \\
\hline
\end{tabular}

Table 1. Hailstone characteristics and terminal velocity. 
By substitute equation (3) and (4) in (2) you can obtain the expression (5):

$$
\frac{R_{\text {hail }} \rho_{\text {hail }}}{\Delta_{\text {hail }}}=\frac{R_{\text {probe }} \rho_{\text {probe }} \text { eq }}{\Delta_{\text {probe }}}
$$

Since hailstone and probe are both spherical it is possible to say that the drag coefficients are equal $\left(\Delta_{\text {hail }}=\right.$ $\Delta_{\text {probe }}$ ). Assuming that it is desired to realize the probe with its equivalent density $\rho_{\text {probe }}=0.1 \mathrm{~g} / \mathrm{cm}^{3}$, considering hail density $\rho_{\text {hail }}=0.87 \mathrm{~g} / \mathrm{cm}^{3}$ and radius of the hailstone $R_{\text {hail }}=4 \mathrm{~mm}$, by solving equation (5) you obtain that youhave to realize a probe with a radius of $R_{\text {probe }}=3.5 \mathrm{~cm}$. According to formula (4), such probe has an equivalent mass $m_{\text {probe }}=18 \mathrm{~g}$.

It is therefore demonstrated the theoretical feasibility of an artificial probe with reasonable weight and size. Such probe will have a terminal velocity $V_{\text {probe }}$ equal to about $45 \mathrm{~km} / \mathrm{h}$, which is in the range of the hailstones terminal velocities reported in Table 1 , thus respecting the preliminary constraint to have similar fluid-dynamic characteristics of hailstones.

\section{Hail Sensing Probes: Practical Realization}

In this section all the aspects of their practical realization are examined.

\subsection{Outer Shell}

The outer shell must be able to withstand the weather conditions inside the cumulonimbus where the hailstones formation occurs. Moreover it must be strong enough to prevent damage to the internal electronics during the possible impact of the probe with "real" hailstones. It is necessary that such container is water-repellent and waterproof, to prevent water and moisture damage the electronics. To meet with these requirements, it can be realized with laminate resin or HDPE (High Density Polyethylene Molecular), in order to create spherical shells with desired radius and a mass of $2.5 \mathrm{~g}$. If the target total mass of the probe is $10 \mathrm{~g}$ or $15 \mathrm{~g}$ (a little lower than what reported in the section 3 to have some more free degrees for manufacturers), we still have available $7.5 \mathrm{~g}$ or $12.5 \mathrm{~g}$, respectively, for all the necessary equipment. In addition, the shell should be able to resist at the variation of the atmospheric pressure with altitude. It could be drilled or equipped with light weight valves.

To realize the outer shell it is necessary to realize two semi-spherical containers. After placing on them all the "intelligence" and the power supply of the probe, we can choose two possible solutions to close the container. At first, it is possible to close the probe by using screws. This solution allows you to re-open the balls, turn on the electronic circuits of the probe, replace the battery and perform maintenance on the sensors, if not directly replace them in case of proven failure. The other possibility is to thermally weld the two semi-spheres and activate the electronics of the probe with a short range electrical charge. This method makes the hail sensing probes more robust but does not allow users to do maintenance operations on them.

\subsection{Sensors}

Different sensors can be installed on the probe. It can be also designed as a multipurpose electronic board where it is possible to change the set of controlled sensors by simply varying the firmware, as it is reported for instance in [15]. To monitor the hail formation process, a set of useful sensor could be the following:

- Triaxial accelerometer.

- Temperature sensor.

- Pressure sensor.

- Humidity sensor.

Together with the PCB (better if realized with wearable sensor materials to keep it light), the antenna required for the data transmission to the receiver and the flash memory for the storage of the data measured by the sensors, these sensors must necessarily have a total weight lower than $6.3 \mathrm{~g}$, if the maximum mass $m_{\text {probe }}=10 \mathrm{~g}$ is considered, or lower than $11.3 \mathrm{~g}$ if the final probe can have a mass up to $m_{\text {probe }}=15 \mathrm{~g}$.

Thanks to the flash memory, the data measured by sensors can be temporarily stored. They are then transmitted to the receiver in bursts, to minimize the power consumption of the probe.

Depending on the monitoring needs, each sensing probe can be equipped with all sensors simultaneously or only with some of them. Moreover, you can note that a GPS receiver is not considered among the set of useful 
sensors. In fact, in this context it is not too important to accurately determine the position of each probe but just their trajectory. The position can be determined with a sufficient precision using the pressure measurements and the integration of the accelerometer measurements.

According to the analysis in section 3, the terminal velocity of the probes is $V_{\text {probe }}=45 \mathrm{~km} / \mathrm{h}$ corresponds to $V_{\text {probe }}=12 \mathrm{~m} / \mathrm{s}$. Since it is roughly possible to estimate a variation of $1{ }^{\circ} \mathrm{C}$ every $100 \mathrm{~m}$ of variation in altitude in free air, it is necessary that the temperature sensor has a sampling period of at least of $10 \mathrm{~s}$. In order to carry out a more refined temperature sampling, we assume that such sampling period can be red used to $T S_{\text {temperature }}=2 \mathrm{~s}$. Humidity and pressure sensors can also work with the same sampling period $\left(T S_{\text {pressure }}=2 \mathrm{~s}, T S_{\text {humidity }}=2 \mathrm{~s}\right)$ in order to have a set of measurements referred at the same time instant. This is very useful for post processing operations when data can be correlated each other.

Triaxial accelerometers data are necessary to monitor the speed and direction changes of the hailstones and to extract their trajectory. The accelerometer needs a sample rate higher than other sensors. Due to the gravity acceleration, the terminal speed $V_{\text {hail }}=45 \mathrm{~km} / \mathrm{h}$ is reached in a time equal to $T_{\text {Vhail }}=1.22 \mathrm{~s}$. Therefore, in order to detect the changes in acceleration with good accuracy, the accelerometer needs a sampling period of $T S_{\text {accelerometer }}$ $=0.1 \mathrm{~s}$.

Let us now suppose that each measured data is coded with 4 bytes. We have 12 bytes of data every 2 seconds due to temperature, pressure and humidity sensors, and 12 bytes each 0.1 seconds due to triaxial accelerometer (4 for each coordinate axes). That means that a single hail sensing probe stores $n$ Byte $=3780$ bytes each 30 seconds, and then transmits them to the receiver.

\subsection{Data Transmission}

In order to assure a good transmission quality, the frequency $F_{T X}=350 \mathrm{MHz}$ would be the best choice, since it is not affected by the presence of hail and water in the atmosphere [16]. But respecting the international laws for RFID and DASH7 systems (as the one proposed here), the best operative frequency that can be used is $F_{T X}=$ $433 \mathrm{MHz}$. The loss introduced into the systems is only $2 \mathrm{~dB}$.

A common commercial transceiver that can be installed on board can have the following characteristics:

- Single dipole antenna.

- Antenna gain, $G_{T X}=2.15 \mathrm{~dB}$.

- Receiver sensitivity, $P_{R X}=-70 \mathrm{dBm}$.

Let us consider also a single receiver, located on the ground and made up by a specific designed electronic interface (which include also RF section) connected to a PC. It is devoted to receive data coming from all the probes and to properly store them in well-designed database. Characteristic of such equipment are:

- Antenna gain $G_{R X}=10 \mathrm{~dB}$.

- Due to antenna gain, the HPBW (Half Power Beam Width) is approximately equal to $60^{\circ}$ : it is large enough to receive the signals from the set of directions from which the probes are transmitting.

- The probes are dropped from a maximum height $h=10,000 \mathrm{~m}$ but it is necessary that the receiver is able to receive signal sup to a distance $r=20,000 \mathrm{~m}$.

- Sensitivity of receiver $P_{R X}=-130 \mathrm{dBm}$.

According to such specifications, by inverting the Fri is equation (6) it is possible to evaluate the minimum power that the probes and the ground receiver must transmit.

$$
P_{R X}=P_{T X} G_{T X} G_{R X}\left(\frac{c}{4 \pi r F_{T X}}\right)^{2}
$$

Each probe must transmits a minimum power of $P_{\text {TXprobe }}=-30 \mathrm{dBm}$, while the ground transceiver must transmit a power $P_{\text {Txground }}=30 \mathrm{dBm}(1 \mathrm{~W})$. The most critical value is $P_{\text {TXprobe }}$ since the probe is powered by a battery which has, of course, a limited duration. Particular attention must be paid to lower as much as possible the probe power consumption. On the other hand, the ground station can be connected to electrical power supply, thus avoiding the implementation of power saving techniques and allowing transmitting $1 \mathrm{~W}$ without problems.

In order to assure that all the artificial hailstones transmit their data to the ground station, the ground receiver performs a polling technique. The ground station knows the amount of fallen probes (almost twenty probes simultaneously). When a probe recognizes its unique identification code (ID) in the inquiring message, it transmits a series of packets to the receiver. The receiver parses the packets and stores the data. 
In order to protect data from errors CRC (Cyclic Redundancy Check) codes can be used, since commercial radiofrequency transceiver chips usually have a native implementation of such techniques.

Given the low amount of data to be transmitted from each probe to the receiver, it is sufficient a simple FSK modulation (Frequency Shift Keying), even better, if it is possible, in its GFSK (Gaussian Frequency Shift Keying) variation, to smooth the transition between the logic levels and limit the spectral bandwidth.

Even the data transmission rate can be low: a speed of $V_{\text {tras }}=4800$ baud is enough and guarantees a good signal to noise ratio together with low bit error rate.

\subsection{Energy Balance}

As already written before, a theoretical terminal velocity of hail sensing probes $V_{\text {probe }}=45 \mathrm{~km} / \mathrm{h}$ is considered together with the as sumption to release them from a maximum height $h=10,000 \mathrm{~m}$. To reach the ground they last about 13 minutes. Since during the whole fall time it is fundamental to guarantee the fully probe operations, it is necessary to use a battery with a capacity sufficient to ensure a sensor average power consumption equal to $I_{\text {sensors }}=100 \mathrm{~mA}$. This value of current consumption is significantly overestimated, but allows evaluating the energy balance in the worst case. Of course it is also necessary that the battery works at the same voltage of the sensors: $3 \mathrm{~V}$.

The terminal speed $V_{\text {probe }}$, does not take account of neither the initial acceleration transitory, nor the terminal velocities at different heights (which are of course different depending on the altitude), nor the possible presence of cross winds upward: all this effects can significantly increase the permanence time in the air of the probe. Therefore it is necessary to considered that the battery life should be sufficient to ensure the power supply to the sensors and to the transceiver for the whole time of the probe "flight" which can be overestimated in $T_{\text {fall }}=20$ minutes.

On the basis of the previous considerations, the necessary energy that has to be available at the battery must be at least $E_{a v}=360 \mathrm{~J}$.

The needed energy can be provided by a common button battery model IEC-CR1616 [17] with the specification reported in Table 2. The CR1616 battery provides $594 \mathrm{~J}$ as evaluated with expression (7).

$$
E_{\text {battery }}=55 \mathrm{mAh} \cdot 3 \mathrm{~V} \cdot 60 \mathrm{~min} \cdot 60 \mathrm{~s}=594 \mathrm{~J}
$$

The commercial sensors with the current consumption reported in

Table 3 require almost $1 \mathrm{~J}$ overall to make the measurements. Of course, when they are not sampling, a specific firmware must keep them in low power consumption mode.

Table 3 reports also the power consumption to transmit data do the ground receiver. It is demonstrated that the battery in Table 2 provide the energy to guarantee that a probe works properly during the fall, thus allowing a good monitoring operations of hail dynamics and behavior during its formation and precipitation to the ground.

\section{Conclusions and Outlooks}

This work presents the feasibility analysis of artificial hailstone to monitor hail formation and behavior. Such hail sensing probes can be designed with similar fluid-dynamics properties of the hailstones and can be realized in practice to increase the type of instrumentations for the remote sensing, the study and the research about hail.

Table 4 reports a summary of the characteristics of the hail sensing probes obtained with the presented work.

Table 2. Technical specification for a battery IEC-CR1616 [17].

\begin{tabular}{cc}
\hline Typical capacity & $55 \mathrm{mAh}$ \\
\hline Chemical System & Lithium/Manganese Dioxide $\left(\mathrm{Li} / \mathrm{MnO}_{2}\right)$ \\
Voltage & $3 \mathrm{~V}$ \\
Typical Weight & $1.1 \mathrm{~g}$ \\
Operative Temperature & $-30^{\circ} \mathrm{C} / 70^{\circ} \mathrm{C}$ \\
Diameter & $16 \mathrm{~mm}$ \\
\hline
\end{tabular}


Table 3. Power consumptionduring the activities made by a single hail sensing probe.

\begin{tabular}{cccc}
\hline Operation & Consumption & Time & Number of activities during $T_{\text {fall }}=20 \mathrm{~min}$ \\
\hline Data Transmission to ground receiver & $-30 \mathrm{dBm}$ & $1 \mathrm{~s}$ & 40 (1 transmission every 30 seconds $)$ \\
Tranceiver consumption while it is on & $3 \mathrm{~mA}$ & $1 \mathrm{~s}$ & 40 (1 every 30 seconds) \\
Accelerometer sensor measure & $0.15 \mathrm{~mA}$ & $10 \mathrm{~ms}$ & $12,000$ (1 every $0.1 \mathrm{~s})$ \\
Temperature sensor measure & $3.6 \mathrm{~mA}$ & $10 \mathrm{~ms}$ & $600(1$ every $2 \mathrm{~s})$ \\
Pressure sensor measure & $3 \mathrm{~mA}$ & $10 \mathrm{~ms}$ & 600 (1 every $2 \mathrm{~s})$ \\
Humidity sensor measure & $1 \mathrm{~mA}$ & $10 \mathrm{~ms}$ & 600 (1 every $2 \mathrm{~s})$ \\
Sensors in stand by (low power mode) & $120 \mu \mathrm{A}$ & & Remaining time during $T_{\text {fall }}$ \\
\hline
\end{tabular}

Table 4. Summary of main characterisctics of the hail sensing probe.

\begin{tabular}{|c|c|}
\hline Ray of the probes (spherical) & $35 \mathrm{~mm}$ \\
\hline Weigth & $10-15 \mathrm{~g}$ \\
\hline Outer shell & Laminate resin or HDPE (High Density Polyethylene Molecular) \\
\hline Terminal velocity on the ground & $36-45 \mathrm{~km} / \mathrm{h}$ \\
\hline Time to fall on the ground from $10,000 \mathrm{~m}$ & 13 - 20 minuti \\
\hline Sensors Voltage & $3 \mathrm{~V}$ \\
\hline Probe meanpowerconsumption & $100 \mathrm{~mA}$ \\
\hline Sensors & Triaxial accelerometer, temperature, pressure, humidiy \\
\hline $\begin{array}{l}\text { Sampling period of the sensors. } \\
\text { Temperature: } \\
\text { Pressure: } \\
\text { Humidity } \\
\text { Triaxial accelerometer }\end{array}$ & $\begin{array}{l}2 \mathrm{~s} \\
2 \mathrm{~s} \\
2 \mathrm{~s} \\
0.1 \mathrm{~s}\end{array}$ \\
\hline Transmissionfrequency & $350 \mathrm{MHz}-433 \mathrm{MHz}$ \\
\hline $\begin{array}{l}\text { Probe RF transceiver } \\
\text { Transmittedpower } \\
\text { Sensitivity }\end{array}$ & $\begin{array}{l}-30 \mathrm{dBm} \\
-70 \mathrm{dBm}\end{array}$ \\
\hline $\begin{array}{c}\text { Ground receiver RF transceiver }{ }^{\mathrm{a}} \\
\text { Transmitted power } \\
\text { Sensitivity }\end{array}$ & $\begin{array}{c}30 \mathrm{dBm} \\
-130 \mathrm{dBm}\end{array}$ \\
\hline Modulationscheme & GFSK \\
\hline Transmissionspeed & 4800 baud \\
\hline Inquiringtechnique & Polling \\
\hline
\end{tabular}

${ }^{\mathrm{a}}$ Not necessary for probe realization but it is needed to evaluate the power link budget and to design the probe transceiver.

The choice of the components and the design of the PCB should be made paying attention to their reliability and robustness, especially when working in harsh environmental conditions.

Of course, some further optimizations can be done during potential future developments. Since the objective is to study the hail and its formation, it is important that the probes are always more similar possible to real hailstones. The idea is therefore to develop probes always smaller with all the necessary electronic equipment inside them and to have probes with different sizes. In order to prevent the probes to fall down to the ground too fast it can be necessary to modify its surface by introducing some protrusion. In this way, calibrating appropriately the mass of the probe, the contact surface and their volume, it is possible to realize artificial hailstones that have the same terminal velocity of the real hail with different and reduces size and even with different shapes.

In the future, in addition to build a lab-demonstration version of the device, and realize some software simulations of the probes behavior during hailstorm, it will be studied in detail how these sensors can be deployed, 
considering the capability to know a priori and forecast where such a hailstorm is going to occur, and how much advanced notice would be needed to deploy them (e.g. to take off in time the UAV or the small plane).

After such studies and simulations, if the probes could be practically realized it will be important to consider also the safety aspects of deploying a larger number of free-falling spherical sensors that physically mimic destructive hailstones. The problem is not addressed in this work since it is only a feasibility analysis of the probes themselves. It will be necessary to understand how and when these sensors can be deployed without damaging property and/or people.

\section{Acknowledgements}

The present study is the result of a research project between the local unit of Consorzio Inter universitario Nazionale per la Fisicadelle Atmosferee delle Idrosfere (CINFAI) within the Department of Electronics and Telecommunications (DET) at Politecnico di Torino and the small enterprise ALMA Ingegneria s.r.l. The project was called "HaSP.—Hail Sensing Probes" and was funded by the Regione Piemonte, Italy, during the year 2015.

\section{References}

[1] Aydin, K., Seliga, T.A. and Balaji, V. (1986) Remote Sensing of Hail with Dual Linear Polarization Radar. J. Climate Appl. Meter., 25, 1475-1484. http://dx.doi.org/10.1175/1520-0450(1986)025<1475:RSOHWA>2.0.CO;2

[2] Fisher, R.E. (1969) Remote Sensing of Hail and Hail Growth in Convective Clouds. Atmospheric Sciences Paper N. 141, Colorado State University, Fort Collins.

[3] Foote, G.B. and Knight, C.A. (1977) Hail: A Review of Hail Science and Hail Suppression. Vol. 16, American Meteorological Society, Boston.

[4] Löffler-Mang, M., Schön, D. and Landry, M. (2011) Characteristics of a New Automatic Hail Recorder. Atmospheric Research, 100, 439-446. http://dx.doi.org/10.1016/j.atmosres.2010.10.026.

[5] Geddes, J.J., Zimmerman, T.A., Wintz, Paul A., Schroeder, T.A., Agee, E.M. and Schmidt, D.K. (1970) Preliminary Design of a Tornado Probe. IEEE Transactions on Geoscience Electronics, 8, 279-284. http://dx.doi.org:/10.1109/TGE.1970.271423

[6] Open Source Tornado Probe Idea \& Concept, Weather Research Technologies. http://www.wxopensource.org/

[7] American Meteorological Society (2009) Glossary of Meteorology. American Meteorological Society, Boston.

[8] Wolf, P. (2003) Meso-Analyst Severe Weather Guide. University Corporation for Atmospheric Research, Boulder.

[9] List, R. (1958) Kennzeichen atmosphärischer Eispartikeln. Zeitschrift für angewandte Mathematik und Physik ZAMP, 10, 180-192. http://dx.doi.org/10.1007/BF02033027

[10] Braham Jr., R.R. (1963) Some Measurement of Snow Pellet Bulk Densities. Journal of Applied Meteorology, 2, 498500. http://dx.doi.org/10.1175/1520-0450(1963)002<0498:SMOSPB >2.0.CO;2

[11] Prodi, F. (1970) Measurements of Local Density in Artificial and Natural Hailstones. Journal of Applied Meteorology, 9, 903-910. http://dx.doi.org/10.1175/1520-0450(1970)009<0903:MOLDIA>2.0.CO;2

[12] List, R., Cantin, J.G. and Ferland, M.G. (1970) Structural Properties of Two Hailstone Samples. Journal of the Atmospheric Sciences, 22, 1080-1090. http://dx.doi.org/10.1175/1520-0469(1970)027<1080:SPOTHS>2.0.CO;2

[13] Knight, N.C. and Heymsfield, A.J. (1983) Measurement and Interpretation of Hailstone Density and Terminal Velocity. Journal of the Atmospheric Sciences, 40, 1510-1516. http://dx.doi.org/10.1175/1520-0469(1983)040<1510:MAIOHD>2.0.CO;2

[14] Castellano, N. E., Nasello, O. B. and Levi, L. (2002) Study of Hail Density Parametrizations. Quarterly Journal of the Royal Meteorological Society, 128, 1445-1460. http://dx.doi.org/10.1002/qi.200212858303

[15] Rorato, O., Lucianaz, C., Bertoldo, S., Allegretti, M. and Perona, G. (2012) A Multipurpose Node for Low Cost Wireless Sensor Network. Proceedings of IEEE-APS Topical Conference on Antennas and Propagation in Wireless Communications (APWC), Cape Town, 2-7 September 2012, 247-250. http://dx.doi.org/10.1109/APWC.2012.6324911

[16] Karmakar, P.K. (2011) Microwave Propagation and Remote Sensing: Atmospheric Influences with Models and Applications. CRC Press, Boca Raton.

[17] Energizer CR1616 Datasheet. http://data.energizer.com/PDFs/cr1616.pdf 